\title{
Noncommutative Theories and Gravity
}

\author{
Victor O. Rivelles* \\ Instituto de Física \\ Universidade de São Paulo \\ Caixa Postal 66318, 05315-970, São Paulo, SP, Brazil \\ E-mail: rivelles@fma.if.usp.br
}

\begin{abstract}
We review the connection between noncommutative field theories and gravity. When the noncommutativity is induced by the Moyal product we can use the Seiberg-Witten map in order to deal with ordinary fields. We then show that the effect of the noncommutativity is the same as a field dependent gravitational background. The gravitational background is that of a gravitational plane wave and the coupling is charge dependent. Uncharged fields couple more strongly than the charged ones. Deviations from the usual dispersion relations are discussed and we show that they are also charge dependent.
\end{abstract}

Fourth International Winter Conference on Mathematical Methods in Physics 09 - 13 August 2004

Centro Brasileiro de Pesquisas Fisicas (CBPF/MCT), Rio de Janeiro, Brazil

\footnotetext{
${ }^{*}$ Speaker.
} 
Noncommutative (NC) theories have a long history [1]. More recently they were found in a limit of string theory with D-branes in a constant NS-NS background B field [2]. In this limit gravity decouples but still leaves a trace in the emerging NC gauge theory. If point splitting regularization is used in the world-sheet then NC appears through the Moyal product which is defined as

$$
A(x) \star B(x)=\left.e^{\frac{i}{2} \theta^{\mu \nu} \partial_{\mu}^{z} \partial_{v}^{y}} A(x) B(y)\right|_{y \rightarrow x},
$$

where $\theta^{\mu \nu}$ is the NC parameter. If Pauli-Villars regularization is used instead then ordinary gauge symmetry is preserved and NC appears in higher dimension operators. Since the S matrix must be regularization independent these two descriptions are related by a space-time field redefinition known as the Seiberg-Witten (SW) map [2].

From the space-time point of view these two descriptions have quite different properties. When the Moyal product is used the ultraviolet structure of the theory is not modified [3] but new infrared divergences appear and get mixed with the ultraviolet ones [4]. This mixing of divergences turns the theory non-renormalizable except in some cases where supersymmetry is present [5]. An important property of $\mathrm{NC}$ theories induced by the Moyal product, which distinguishes them from the conventional ones, is that translations in the NC directions are equivalent to gauge transformations [6]. This can be seen even for the case of a scalar field which has the NC gauge transformation $\delta \hat{\phi}=-i[\hat{\phi}, \hat{\lambda}]_{\star}$, where $[A, B]_{\star}=A \star B-B \star A$ is the Moyal commutator. Under a global translation the scalar field transforms as $\delta_{T} \phi=\xi^{\mu} \partial_{\mu} \hat{\phi}$. Derivatives of the field can be rewritten using the Moyal commutator as $\partial_{\mu} \hat{\phi}=-i \theta_{\mu \nu}^{-1}\left[x^{v}, \hat{\phi}\right]_{\star}$ so that $\delta \hat{\phi}=\delta_{T} \hat{\phi}$ with gauge parameter $\hat{\lambda}=-\theta_{\mu \nu}^{-1} \xi^{\mu} x^{\nu}$. The only other field theory which has a similar property is general relativity where local translations are gauge transformations associated to general coordinate transformations. This remarkable property shows that, as in general relativity, there are no local gauge invariant observables in NC theories.

In the description obtained through the SW map the theory is presented as a series expansion in $\theta$. In this way a local field theory is obtained at the expense of introducing a large number of non-renormalizable interactions [7]. At the classical level, on the other side, it is possible to understand very clearly the breakdown of Lorentz invariance induced by the noncommutativity. The dispersion relation for plane waves in a magnetic background gets modified so that photons do not move with the velocity of light [8]. However, the connection between translations and gauge transformations seems to be lost. A global translation on a commutative real scalar field $\delta_{T} \phi=\xi^{\mu} \partial_{\mu} \phi$ can no no longer be rewritten as a gauge transformation since $\delta \phi=0$. We will discuss how other aspects concerning gravity emerges in the description which makes use of the $\mathrm{SW}$ map. In this case NC field theories can be interpreted as ordinary theories immersed in a gravitational background generated by the gauge field. We will show that the $\theta$ dependent terms in the commutative action can be interpreted as a gravitational background which depends on the gauge field. We then determine the metric which couples to real and complex scalar fields. We find that the uncharged field coupling is twice that of the charged one. So we can interpret the gauge coupling in NC theory as a particular gravitational coupling which depends on the charge of the field. We also determine the geodesics followed by a massless particle in this background. We find that its velocity differs from the velocity of light by an amount proportional to $\theta$ with the deviation for the uncharged case being twice that of the charged one. For the uncharged case the deviation is the same as that found for the the gauge theory in flat space-time $[8,9]$. As a final check we derive 
these same velocities in a field theoretic context.

The action for the NC Abelian gauge theory in flat space-time is

$$
S_{A}=-\frac{1}{4} \int d^{4} x \hat{F}^{\mu v} \star \hat{F}_{\mu v}
$$

where $\hat{F}_{\mu v}=\partial_{\mu} \hat{A}_{v}-\partial_{v} \hat{A}_{\mu}-i\left[\hat{A}_{\mu}, \hat{A}_{v}\right]_{\star}$. For a real scalar field in the adjoint representation of $U(1)$ the flat space-time action is

$$
S_{\varphi}=\frac{1}{2} \int d^{4} x \hat{D}^{\mu} \hat{\varphi} \star \hat{D}_{\mu} \hat{\varphi}
$$

where $\hat{D}_{\mu} \hat{\varphi}=\partial_{\mu} \hat{\varphi}-i\left[\hat{A}_{\mu}, \hat{\varphi}\right]_{\star}$. On the other side, for a complex scalar field in the fundamental representation of $U(1)$ the action is

$$
S_{\phi}=\int d^{4} x \hat{D}^{\mu} \hat{\phi} \star\left(\hat{D}_{\mu} \hat{\phi}\right)^{\dagger}
$$

with $\hat{D}_{\mu} \hat{\phi}=\partial_{\mu} \hat{\phi}-i \hat{A}_{\mu} \star \hat{\phi}$. The gauge transformations which leave the above actions invariant are given by

$$
\delta \hat{A}_{\mu}=\hat{D}_{\mu} \hat{\lambda}, \quad \delta \hat{\varphi}=-i[\hat{\varphi}, \hat{\lambda}]_{\star}, \quad \delta \hat{\phi}=i \hat{\lambda} \star \hat{\phi}, \quad \delta \hat{\phi}^{\dagger}=-i \hat{\phi}^{\dagger} \star \hat{\lambda} .
$$

To first order in $\theta$ the SW map is $[2,10]$

$$
\begin{aligned}
\hat{A}_{\mu} & =A_{\mu}-\frac{1}{2} \theta^{\alpha \beta} A_{\alpha}\left(\partial_{\beta} A_{\mu}+F_{\beta \mu}\right), \\
\hat{\varphi} & =\varphi-\theta^{\alpha \beta} A_{\alpha} \partial_{\beta} \varphi, \\
\hat{\phi} & =\phi-\frac{1}{2} \theta^{\alpha \beta} A_{\alpha} \partial_{\beta} \phi,
\end{aligned}
$$

We can now expand the NC actions (2),(3) and (4) using (1) and apply the map (6) to get the corresponding commutative actions.

For the real scalar field we find, always to first order in $\theta$,

$$
S_{\varphi}=\frac{1}{2} \int d^{4} x\left[\partial^{\mu} \varphi \partial_{\mu} \varphi+2 \theta^{\mu \alpha} F_{\alpha}{ }^{\nu}\left(-\partial_{\mu} \varphi \partial_{\nu} \varphi+\frac{1}{4} \eta_{\mu \nu} \partial^{\rho} \varphi \partial_{\rho} \varphi\right)\right]
$$

It is worth to remark that the tensor inside the parenthesis is traceless. If we now consider this same field coupled to a gravitational background

$$
S_{g, \varphi}=\frac{1}{2} \int d^{4} x \sqrt{-g} g^{\mu v} \partial_{\mu} \varphi \partial_{v} \varphi,
$$

and expand the metric $g_{\mu v}$ around the flat metric $\eta_{\mu v}$,

$$
g_{\mu v}=\eta_{\mu v}+h_{\mu v}+\eta_{\mu v} h
$$

where $h_{\mu v}$ is traceless, we get

$$
S_{g, \varphi}=\frac{1}{2} \int d^{4} x\left(\partial^{\mu} \varphi \partial_{\mu} \varphi-h^{\mu \nu} \partial_{\mu} \varphi \partial_{\nu} \varphi+h \partial^{\rho} \varphi \partial_{\rho} \varphi\right),
$$


where indices are raised and lowered with the flat metric. Since both actions, (7) and (10), have the same structure we can identify a linearized background gravitational field

$$
\begin{aligned}
h^{\mu \nu} & =\theta^{\mu \alpha} F_{\alpha}{ }^{v}+\theta^{v \alpha} F_{\alpha}{ }^{\mu}+\frac{1}{2} \eta^{\mu v} \theta^{\alpha \beta} F_{\alpha \beta}, \\
h & =0 .
\end{aligned}
$$

Then, the effect of noncommutativity on the commutative scalar field is similar to a field dependent gravitational field [10].

The same procedure can be repeated for the complex scalar field [10]. We find the linearized metric

$$
\begin{aligned}
h^{\mu v} & =\frac{1}{2}\left(\theta^{\mu \alpha} F_{\alpha}{ }^{v}+\theta^{v \alpha} F_{\alpha}{ }^{\mu}\right)+\frac{1}{4} \eta^{\mu v} \theta^{\alpha \beta} F_{\alpha \beta}, \\
h & =0 .
\end{aligned}
$$

Then charged fields feel a gravitational background which is half of that felt by the uncharged ones. Therefore, the gravity coupling is now dependent on the charge of the field, being stronger for uncharged fields.

Notice that the gauge field has now a dual role, it couples minimally to the charged field and also as a gravitational background. As it is well known the SW map gives rise to the following action

$$
S_{A}=-\frac{1}{4} \int d^{4} x\left[F^{\mu v} F_{\mu v}+2 \theta^{\mu \rho} F_{\rho}{ }^{v}\left(F_{\mu}{ }^{\sigma} F_{\sigma v}+\frac{1}{4} \eta_{\mu v} F^{\alpha \beta} F_{\alpha \beta}\right)\right] .
$$

Again, the tensor inside the parenthesis is traceless. At this point we could be tempted to consider this action as some gravitational action build up from the metric (11) or (12). Since the field strength always appears multiplied by $\theta$ inside the metric, all invariants constructed with it will be of order $\theta$. Hence, they can not give rise to (13), unless they appear in combinations involving the inverse of $\theta$. If we insist in having an action which is polynomial in $\theta$ the best we can do is to regard the gauge field as having a double role again and couple it to gravitation as in the previous case. We then find that $h^{\mu \nu}$ is given by (12). Since the NC gauge field resembles a non-Abelian gauge field we expect that its commutative counterpart couple to the same gravitational field as the charged one. It should also be remarked that in this case the gravitational field can not be interpreted just as a fixed background since it depends on the dynamical gauge field.

Having determined the field dependent background metric we can now study its properties. A detailed analysis shows that it describes a plane gravitational wave [10].

We can now turn our attention to the behavior of a massless particle in this background. Its geodesics is described by

$$
d s^{2}=\left(1+\frac{1}{4} \theta^{\alpha \beta} F_{\alpha \beta}\right) d x^{\mu} d x_{\mu}+\theta_{\mu \alpha} F_{v}^{\alpha} d x^{\mu} d x^{v}=0 .
$$

If we consider the case where there is no noncommutativity between space and time, that is $\theta^{0 i}=0$, and calling $\theta^{i j}=\varepsilon^{i j k} \theta^{k}, F^{i 0}=E^{i}$, and $F^{i j}=\varepsilon^{i j k} B^{k}$, we find to first order in $\theta$ that

$$
\left(1-\vec{v}^{2}\right)(1-2 \vec{\theta} \cdot \vec{B})-\vec{\theta} \cdot(\vec{v} \times \vec{E})+\vec{v}^{2} \vec{\theta} \cdot \vec{B}-(\vec{B} \cdot \vec{v})(\vec{\theta} \cdot \vec{v})=0,
$$


where $\vec{v}$ is the particle velocity. Then to zeroth order, the velocity $\vec{v}_{0}$ satisfies $\vec{v}_{0}^{2}=1$ as it should. We can now decompose all vectors into their transversal and longitudinal components with respect to $\vec{v}_{0}, \vec{E}=\vec{E}_{T}+\vec{v}_{0} E_{L}, \vec{B}=\vec{B}_{T}+\vec{v}_{0} B_{L}$ and $\vec{\theta}=\vec{\theta}_{T}+\vec{v}_{0} \theta_{L}$. We then find that the velocity is

$$
\vec{v}^{2}=1+\vec{\theta}_{T} \cdot\left(\vec{B}_{T}-\vec{v}_{0} \times \vec{E}_{T}\right)
$$

Hence, a charged massless particle has its velocity changed with respect to the velocity of light by an amount which depends on $\theta$. For an uncharged massless particle

$$
\vec{v}^{2}=1+2 \vec{\theta}_{T} \cdot\left(\vec{B}_{T}-\vec{v}_{0} \times \vec{E}_{T}\right),
$$

and the correction due to the noncommutativity is twice that of a charged particle.

We can now check the consistency of these results by going back to the original action (7) and computing the group velocity for planes waves. Upon quantization they give the velocity of the particle associated to the respective field. For the uncharged scalar field we get the equation of motion

$$
\left(1-\frac{1}{2} \theta^{\mu v} F_{\mu v}\right) \square \varphi-2 \theta^{\mu \alpha} F_{\alpha}{ }^{v} \partial_{\mu} \partial_{\nu} \varphi=0 .
$$

If the field strength is constant we can find a plane wave solution with the following dispersion relation

$$
\left(1-\frac{1}{2} \theta^{\mu v} F_{\mu \nu}\right) k^{2}-2 \theta^{\mu \alpha} F_{\alpha}{ }^{v} k_{\mu} k_{v}=0,
$$

and using the same conventions for vectors as before, it results in

$$
\frac{\vec{k}^{2}}{\omega^{2}}=1-2 \vec{\theta}_{T} \cdot\left(\vec{B}_{T}-\frac{\vec{k}}{\omega} \times \vec{E}_{T}\right),
$$

where $k^{\mu}=(\omega, \vec{k})$. We then find that the phase and group velocities coincide and are given by (17) as expected. For the charged scalar field we have to turn off the gauge coupling in order to get a plane wave solution. In this case the equation of motion is

$$
\left(1-\frac{1}{4} \theta^{\mu v} F_{\mu \nu}\right) \square \phi-\theta^{\mu \alpha} F_{\alpha}{ }^{v} \partial_{\mu} \partial_{v} \phi=0 .
$$

In a constant field strength background the dispersion relation for a plane wave reads as in (19) with $\theta$ replaced by $\theta / 2$. Then we must perform the same replacement in the phase and group velocities and we get (16). Therefore, in both pictures, noncommutative and gravitational, we get the same results.

For the gauge field the situation is more subtle because of its double role. There is no clear way to split the action (13). What can be done is to break up the gauge field into a background plus a plane wave as in [8]. We then get the following dispersion relation

$$
k^{2}-2 \theta^{\mu \alpha} F_{\alpha}{ }^{v} k_{\mu} k_{v}=0
$$

where $F_{\alpha}{ }^{v}$ is now the constant background. This leads to (20), that is, the dispersion relation for the uncharged scalar field. It also reproduces the result in $[8,9]$ when the background is purely magnetic. This shows the dual role of the gauge field, since it couples to gravitation as a charged 
field but its dispersion relation is that of an uncharged field. It should be remarked that it is possible to derive the dispersion relation for the general case, including the interesting situation when $\theta^{0 i} \neq$ 0 . A detailed analysis will be presented in [11].

We have seen that it is possible to regard noncommutative theories as conventional theories embedded in a gravitational background produced by the gauge field. This brings a new connection between noncommutativity and gravitation. We could imagine that this is a peculiarity of the first order term in the $\theta$ expansion of the SW map but an analysis to all orders in $\theta$ was performed in [12].

I would like to thank the organizers for the kind invitation to deliver this talk. This work was partially supported by FAPESP, CNPq and PRONEX under contract CNPq 66.2002/1998-99.

\section{References}

[1] For a review on NC field theories see: R. J. Szabo, Quantum field theory on noncommutative spaces, hep-th/0109162; M. R. Douglas and N. A. Nekrasov, Noncommutative field theory, Rev. Mod. Phys. 73 (2001) 977 [hep-th/0106048]; V. O. Rivelles, Noncommutative supersymmetric theories, hep-th/0211169; V. O. Rivelles, Supersymmetry and gravity in noncommutative field theories, Nucl. Phys. Proc. Suppl. B 127 (2004) 63 [hep-th/0305122].

[2] N. Seiberg and E. Witten, String theory and noncommutative geometry, JHEP 9909 (1999) 032 [hep-th/9908142].

[3] T. Filk, Divergences In A Field Theory On Quantum Space, Phys. Lett. B376 (1996) 53.

[4] S. Minwalla, M. Van Raamsdonk and N. Seiberg, Noncommutative perturbative dynamics, JHEP 0002 (2000) 020 [hep-th/9912072].

[5] H. O. Girotti, M. Gomes, V. O. Rivelles and A. J. da Silva, A consistent noncommutative field theory: The Wess-Zumino model, Nucl. Phys. B587 (2000) 299 [hep-th/0005272]; H. O. Girotti, M. Gomes, V. O. Rivelles and A. J. da Silva, The noncommutative supersymmetric nonlinear sigma model, Int. J. Mod. Phys. $A 17$ (2002) 1503 [hep-th/0102101]; H. O. Girotti, M. Gomes, A. Y. Petrov, V. O. Rivelles and A. J. da Silva, The three-dimensional noncommutative nonlinear sigma model in superspace, Phys. Lett. B521 (2001) 119 [hep-th/0109222]; A. F. Ferrari, H. O. Girotti, M. Gomes, A. Y. Petrov, A. A. Ribeiro, V. O. Rivelles and A. J. da Silva, Superfield covariant analysis of the divergence structure of noncommutative supersymmetric QED(4), Phys. Rev. D69 (2004) 025008 [hep-th/0309154]; A. F. Ferrari, H. O. Girotti, M. Gomes, A. Y. Petrov, A. A. Ribeiro, V. O. Rivelles and A. J. da Silva, Towards a consistent noncommutative supersymmetric Yang-Mills theory: Superfield covariant analysis, hep-th/0407040.

[6] D. J. Gross and N. A. Nekrasov, Dynamics of strings in noncommutative gauge theory, JHEP 0010 (2000) 021 [hep-th/0007204].

[7] For a review see: R. Wulkenhaar, Quantum field theories on noncommutative $R * * 4$ versus Theta-expanded quantum field theories, hep-th/0206018.

[8] Z. Guralnik, R. Jackiw, S. Y. Pi and A. P. Polychronakos, Testing non-commutative QED, constructing non-commutative MHD, Phys. Lett. B517 (2001) 450 [hep-th/0106044].

[9] R. G. Cai, Superluminal noncommutative photons, Phys. Lett. B517 (2001) 457 [hep-th/0106047]. 
[10] V. O. Rivelles, Noncommutative field theories and gravity, Phys. Lett. B558 (2003) 191 [hep-th/0212262].

[11] T. Mariz, J. R. Nascimento and V. O. Rivelles, in preparation.

[12] R. Banerjee and H. S. Yang, Exact Seiberg-Witten map, induced gravity and topological invariants in noncommutative field theories, hep-th/0404064; H. S. Yang, Exact Seiberg-Witten map and induced gravity from noncommutativity, hep-th/0402002; R. Banerjee, C. k. Lee and H. S. Yang, Seiberg-Witten-type maps for currents and energy-momentum tensors in noncommutative gauge theories, hep-th/0312103. 